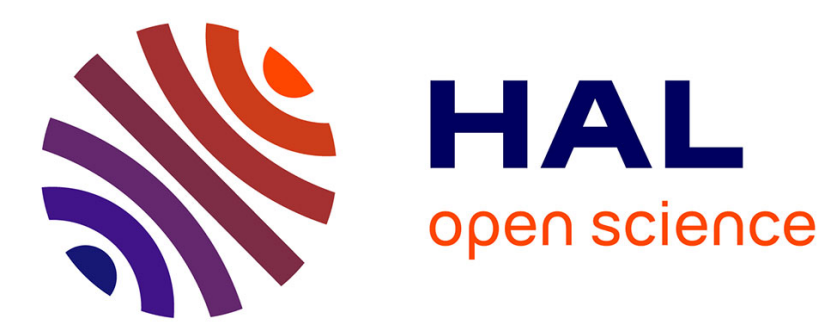

\title{
MRAS-based Sensorless Speed Integral Backstepping Control for Induction Machine, using a Flux Backstepping Observer
}

\author{
Mohamed Horch, Abdelmadjid Boumediene, Lotfi Baghli
}

\section{To cite this version:}

Mohamed Horch, Abdelmadjid Boumediene, Lotfi Baghli. MRAS-based Sensorless Speed Integral Backstepping Control for Induction Machine, using a Flux Backstepping Observer. International Journal of Power Electronics and Drive Systems, 2017, 8 (4), pp.1650 - 1662. 10.11591/ijpeds.v8.i4.pp16501662 . hal-01800495

\section{HAL Id: hal-01800495 \\ https://hal.science/hal-01800495}

Submitted on 29 May 2021

HAL is a multi-disciplinary open access archive for the deposit and dissemination of scientific research documents, whether they are published or not. The documents may come from teaching and research institutions in France or abroad, or from public or private research centers.
L'archive ouverte pluridisciplinaire HAL, est destinée au dépôt et à la diffusion de documents scientifiques de niveau recherche, publiés ou non, émanant des établissements d'enseignement et de recherche français ou étrangers, des laboratoires publics ou privés. 


\title{
MRAS-based Sensorless Speed Integral Backstepping Control for Induction Machine, using a Flux Backstepping Observer
}

\author{
Mohamed Horch ${ }^{1}$, Abdelmadjid Boumediene ${ }^{2}$, Lotfi Baghli \\ ${ }^{1,2,3}$ Université de Tlemcen, LAT, 13000, Tlemcen, Algeria \\ ${ }^{3}$ Université de Lorraine, GREEN, EA 4366F-54500, Vandoeuvre-lès-Nancy, France
}

\section{Article Info \\ Article history: \\ Received Sep 10, 2017 \\ Revised Oct 22, 2017 \\ Accepted Nov 14, 2017 \\ Keyword: \\ Field oriented control Induction machine drive Integral Backstepping MRAS observer Sensorless control}

\begin{abstract}
This paper presents a study concerning a sensorless vector control of an induction machine fed by a voltage source inverter. The aim is to provide a scheme to control the speed and the rotor flux using a sensorless integral backstepping control approach. The rotor speed estimation is done by an observer using the model reference adaptive system (MRAS) technique whereas the nonlinear backstepping observer is used to get the rotor flux. The main objective is to achieve a robust control, adaptive and efficient, which will allow us to test and evaluate the performance of the proposed observer, combined with a sensorless control of the induction machine. Tests and validation are done using numerical simulations with MATLAB/SIMULINK-PSB (Power System Block set) toolbox. The results show good performance in terms of robustness regarding machine parameter variations and show the excellent quality of the control law associated with the observer, despite the observability problems when the machine operates at low speed.
\end{abstract}

Copyright (c) 2017 Institute of Advanced Engineering and Science. All rights reserved.

\section{Corresponding Author:}

Mohamed Horch,

Department of Electrical and Electronic Engineering,

Tlemcen University,

University of Tlemcen, LAT, 13000, Tlemcen, Algeria.

Email: mohamed.horch@mail.univ-tlemcen.dz

\section{INTRODUCTION}

In the recent decades, the variable speed electric drives developed rapidly and aroused significant interest in most sectors of industrial activity implementing the driving force, due to technological advances, but also methodological [1].The direct current machine has long been the best source of electro mechanical variable speed because of its ease of control. This machine, with its two coils to orthogonal fields, presents a natural decoupling between stator flux and electromagnetic torque [2]. However, its major disadvantage is the existence of the "collector-brush" device which is the origin of his employment limitations. This mechanical device is poorly tolerated in some applications for its high price, its frequent and costly maintenance, but also because of the presence of electrical arcs prohibiting use in explosive atmospheres. Due to these constraints, research was carried for other solutions, exploiting the AC machines and especially the squirrel cage induction motors (IM) [3].The IM has certain advantages related to its simple design, robustness, low cost and maintenance. Also, it is widely used in many fields such as aerospace, rail and marine traction, transportation systems and in a multitude of industrial applications (machine tools, driving machines, pumping systems, ventilation and lifting [4].

However, the use of the induction machine for variable speed drives raises a couple of difficulties, which leads to a complex control compared to that of another motor. Indeed, this type of motor is characterized by a multivariable and nonlinear mathematical model, with a strong coupling between the control variables of the magnetic flux and the electromagnetic torque. It is not possible to control a motor 
output variable (torque, speed or position) by varying a single input variable (current or voltage). Moreover, some of its state variables are not accessible to measurement and the motor parameters are subject to variations in time [5], [6].The implementation of these control methods were only possible through the development of digital signal controllers, microcontrollers, static converters. Also, several techniques have been proposed to obtain for the induction machine, the high-performance control devices, and experience its qualities and benefits in the field of variable speed [7].

Among the controls, we distinguish the vector control based on dynamic models, using the Park transformation to solve the torque-flux decoupling issue. The combination of this technique with a modern automatic approaches based on nonlinear control algorithms such as the Backstepping control contribute to solve the robustness problem (the change of load torque and machine parameters). However, it requires the installation of an incremental encoder to measure the rotor speed [8-9]. Using the encoder leads to additional cost which can be higher than the machine one, for low power application. In addition, there must be an additional space for installing the encoder. The system reliability decreases because of this fragile device. Because of all these inconvenient, one tried to exclude the use the incremental encoder and research on the sensorless control of induction machines began. Several strategies were proposed in the literature to achieve this objective [10-11]. Much of the proposed methods are based on observers and estimators in open loop. They largely depend on the model of the induction machine. For this reason, these techniques fail to replace the incremental encoder in the field of low speeds. Further research based on the combination of modern techniques and theories including adaptive laws. These techniques promise to give better results, even in the low-speed including stopped.

In this paper, we chose to use a nonlinear integral backstepping control design scheme, for speed control and we propose a new combination for the realization of robust observers, by using the model reference adaptive system (MRAS) to estimate the rotor speed and the Backstepping observer to estimate the rotor flux.

\section{INDUCTION MOTOR MODEL AND VECTOR CONTROL}

\subsection{Model of IM in (d-q) Reference Frame}

The mathematical model of the induction machine in the $(d-q)$ synchronously rotating frame is given by the following nonlinear equations [12-13]:

$$
\begin{aligned}
& \dot{i}_{s d}=a_{1} \cdot i_{s d}+\omega_{s} \cdot i_{s q}+a_{2} \cdot \varphi_{r d}+a_{3} \omega_{r} \cdot \varphi_{r q}+b \cdot v_{s d} \\
& \dot{i}_{s q}=-\omega_{s} \cdot i_{s d}+a_{1} \cdot i_{s q}-a_{3} \cdot \varphi_{r d} \cdot \omega_{r}+a_{2} \cdot \varphi_{r q}+b \cdot v_{s q} \\
& \dot{\varphi}_{\mathrm{rd}}=\mathrm{a}_{4} \cdot \dot{i}_{\mathrm{sd}}-\mathrm{a}_{5} \cdot \varphi_{\mathrm{rd}}+\left(\omega_{\mathrm{s}}-\omega_{\mathrm{r}}\right) \cdot \varphi_{\mathrm{rq}} \\
& \dot{\varphi}_{\mathrm{rq}}=\mathrm{a}_{4} \cdot \dot{\mathrm{i}}_{\mathrm{sq}}-\left(\omega_{\mathrm{s}}-\omega_{\mathrm{r}}\right) \cdot \varphi_{\mathrm{rd}}-\mathrm{a}_{5} \cdot \varphi_{\mathrm{rq}} \\
& \left.\dot{\omega}_{\mathrm{r}}=\mathrm{a}_{6} \cdot \dot{i}_{\mathrm{sq}} \cdot \varphi_{\mathrm{rd}}-\mathrm{i}_{\mathrm{sd}} \cdot \varphi_{\mathrm{rq}}\right)-\mathrm{a}_{7} \cdot \omega_{\mathrm{r}}-\mathrm{a}_{8} \cdot \mathrm{T}_{\mathrm{l}}
\end{aligned}
$$

With

$$
\begin{aligned}
& a_{1}=-b \cdot\left(R_{s}+\frac{L_{m}^{2}}{L_{r}^{2}} \cdot R_{r}\right), a_{2}=\frac{L_{m} \cdot R_{r}}{\left(\sigma . L_{s} \cdot L_{r}^{2}\right)}, a_{3}=-\frac{L_{m}}{\left(\sigma \cdot L_{s} \cdot L_{r}\right)} \\
& a_{4}=\frac{L_{m} \cdot R_{r}}{L_{r}}, a_{5}=\frac{R_{r}}{L_{r}}, a_{6}=\frac{p^{2} \cdot L_{m}}{J \cdot L_{r}}, a_{7}=\frac{f_{c}}{J}, a_{8}=\frac{p}{J} \\
& \sigma=1-\frac{L_{m}^{2}}{L_{s} \cdot L_{r}}, b=\frac{1}{\sigma \cdot L_{s}}
\end{aligned}
$$

\subsection{Direct rotor flux orientation (DRFO)}

The aim of vector control is to let the induction machine effectively respond to reference variations (rotor position, torque, speed) in a wide range of operation and for applications requiring high dynamic performance. It is based on the instantaneous torque control and a suitable choice of reference (d, q) of Park, so that the d-axis is aligned with the rotor flux vector, to obtain the decoupling between the electromagnetic torque and rotor flux. This alignment imposes the following condition: [14-15]:

$$
\varphi_{\mathrm{r}}=\varphi_{\mathrm{rd}} \text { and } \varphi_{\mathrm{rq}}=0
$$

This choice allows to control the torque via the current $i_{s q}$ and the rotor flux through the current $\mathrm{i}_{\text {sd }}$.

\section{INTEGRAL BACKSTEPPING CONTROL}

MRAS-based Sensorless Speed Integral Backstepping Control for Induction Machine ... (Mohamed Horch) 


\subsection{Principle of the integral backstepping control}

The Backstepping technique is a relatively new control method of nonlinear systems. The principle of this approach is to stabilize at the beginning the first sub system by a stabilizing function known via a chosen Lyapunov function, then to add to its error an integrator. The same procedure for the successive subsystems to finally reach a global Lyapunov function giving the overall control law that stabilizes the system [16-17]. The combination of technical Backstepping with vector control of the induction machine gives the better performance of the control law [18-19].

\subsection{The integral Backstepping control for IM and rotor flux estimator}

The objective to design an integral backstepping control, allowing to ensure the tracking of rotor speed and rotor flux for the machine [20-21], we define the errors $\mathrm{e}_{\omega_{\mathrm{r}}}$ and $\mathrm{e}_{\varphi_{\mathrm{r}}}$ respectively representing the error between the actual speed $\omega_{\mathrm{r}}$ and the reference speed $\omega_{\mathrm{r}}^{*}$ and the error between the flux module $\varphi_{\mathrm{dr}}$ and its reference $\varphi_{\mathrm{dr}}^{*}$.

\section{a) First step "speed and flux loop"}

$$
\begin{aligned}
& \mathrm{e}_{\omega_{\mathrm{r}}}=\omega_{\mathrm{r}}^{*}-\omega_{\mathrm{r}}+\mathrm{z}_{\omega_{\mathrm{r}}} \\
& \mathrm{e}_{\varphi_{\mathrm{rd}}}=\varphi_{\mathrm{rd}}^{*}-\varphi_{\mathrm{rd}}+\mathrm{z}_{\varphi_{\mathrm{rd}}}
\end{aligned}
$$

With:

$$
\begin{aligned}
& \mathrm{z}_{\varphi_{\mathrm{rd}}}=\mathrm{k}_{\varphi_{\mathrm{rd}}}^{\prime} \int_{0}^{\mathrm{t}}\left(\varphi_{\mathrm{rd}}^{*}-\varphi_{\mathrm{rd}}\right) \mathrm{dt} \\
& \mathrm{z}_{\omega_{\mathrm{r}}}=\mathrm{k}_{\varphi_{\mathrm{rd}}}^{\prime} \int_{0}^{\mathrm{t}}\left(\omega_{\mathrm{r}}^{*}-\omega_{\mathrm{r}}\right) \mathrm{dt}
\end{aligned}
$$

Then the dynamical error equations are given by:

$$
\begin{aligned}
& \dot{\mathrm{e}}_{\omega_{\mathrm{r}}}=\dot{\omega}_{\mathrm{r}}^{*}-\dot{\omega}_{\mathrm{r}}+\dot{\mathrm{z}}_{\omega_{\mathrm{r}}} \\
& \dot{\mathrm{e}}_{\varphi_{\mathrm{rd}}}=\dot{\varphi}_{\mathrm{rd}}^{*}-\dot{\varphi}_{\mathrm{rd}}+\dot{\mathrm{z}}_{\varphi_{\mathrm{rd}}}
\end{aligned}
$$

With:

$$
\begin{aligned}
\dot{\mathrm{z}}_{\varphi_{\mathrm{rd}}}=\mathrm{k}_{\varphi_{\mathrm{rd}}}^{\prime}\left(\varphi_{\mathrm{rd}}^{*}-\varphi_{\mathrm{rd}}\right) & \\
\dot{\mathrm{z}}_{\omega_{\mathrm{r}}} & =\mathrm{k}_{\omega_{\mathrm{r}}}^{\prime}\left(\omega_{\mathrm{r}}^{*}-\omega_{\mathrm{r}}\right)
\end{aligned}
$$

Then the error dynamical equations becomes:

$$
\begin{aligned}
& \dot{\mathrm{e}}_{\omega_{\mathrm{r}}}=\dot{\omega}_{\mathrm{r}}^{*}-\mathrm{a}_{6} \cdot\left(\mathrm{i}_{\mathrm{sq}} \cdot \varphi_{\mathrm{rd}}\right)+\mathrm{a}_{7} \cdot \omega_{\mathrm{r}}+\mathrm{a}_{8} \cdot \mathrm{T}_{1}+\dot{\mathrm{z}}_{\omega_{\mathrm{r}}} \\
& \dot{\mathrm{e}}_{\varphi_{\mathrm{dr}}}=\dot{\varphi}_{\mathrm{rd}}^{*}-\mathrm{a}_{4} \cdot \dot{\mathrm{i}}_{\mathrm{sd}}+\mathrm{a}_{5} \cdot \varphi_{\mathrm{rd}}+\dot{\mathrm{z}}_{\varphi_{\mathrm{dr}}}
\end{aligned}
$$

We require that $\dot{\mathrm{e}}_{\omega_{\mathrm{r}}}=-\mathrm{k}_{\omega_{\mathrm{r}}} \mathrm{e}_{\omega_{\mathrm{r}}}$ and $\dot{\mathrm{e}}_{\varphi_{\mathrm{rd}}}=-\mathrm{k}_{\varphi_{\mathrm{rd}}} \mathrm{e}_{\varphi_{\mathrm{rd}}}$, where $\mathrm{k}_{\omega_{\mathrm{r}}}$ and $\mathrm{k}_{\varphi_{\mathrm{dr}}}$ are positives constants.

We can use the Lyapunov function:

$$
\mathrm{V}=\frac{1}{2}\left(\mathrm{e}_{\omega_{\mathrm{r}}}^{2}+\mathrm{e}_{\varphi_{\mathrm{rd}}}^{2}\right)
$$


Its derivative is:

$$
\begin{aligned}
& \dot{\mathrm{V}}=\mathrm{e}_{\omega_{\mathrm{r}}} \dot{\mathrm{e}}_{\omega_{\mathrm{r}}}+\mathrm{e}_{\varphi_{\mathrm{rdd}}} \dot{\mathrm{e}}_{\varphi_{\varphi_{\mathrm{rd}}}} \\
& \dot{\mathrm{V}}=\mathrm{e}_{\omega_{\mathrm{r}}}\left(\dot{\omega}_{\mathrm{r}}^{*}-\mathrm{a}_{6} \cdot\left(\mathrm{i}_{\mathrm{sq}} \cdot \varphi_{\mathrm{rd}}\right)+\mathrm{a}_{7} \cdot \omega_{\mathrm{r}}+\mathrm{a}_{8} \cdot \mathrm{T}_{1}+\dot{\mathrm{z}}_{\omega_{\mathrm{r}}}\right)+\mathrm{e}_{\varphi_{\mathrm{rd}}}\left(\dot{\varphi}_{\mathrm{rd}}^{*}-\mathrm{a}_{4} \cdot \dot{\mathrm{i}}_{\mathrm{sd}}+\mathrm{a}_{5} \cdot \varphi_{\mathrm{rd}}+\dot{\mathrm{z}}_{\varphi_{\mathrm{dr}}}\right)
\end{aligned}
$$

The aim of tracking is fulfilled $(\dot{V}<0)$ choosing the virtual control that represents the stabilization function as follows:

$$
\begin{aligned}
& i_{\mathrm{sq}}^{*}=\frac{1}{\varphi_{\mathrm{rd}} \cdot a_{6}}\left[\mathrm{k}_{\omega_{\mathrm{r}}} \mathrm{e}_{\omega_{\mathrm{r}}}+\omega_{\mathrm{r}}^{*}+\mathrm{a}_{7} \cdot \omega_{\mathrm{r}}+\mathrm{a}_{8} \cdot \mathrm{T}_{\mathrm{l}}+\dot{\mathrm{z}}_{\omega_{\mathrm{r}}}\right] \\
& \mathrm{i}_{\mathrm{sd}}^{*}=\frac{1}{\mathrm{a}_{4}}\left[\mathrm{k}_{\varphi_{\mathrm{rd}}} \mathrm{e}_{\varphi_{\mathrm{rd}}}+\dot{\varphi}_{\mathrm{re}}^{*}+\mathrm{a}_{5} \varphi_{\mathrm{rd}}+\dot{\mathrm{z}}_{\varphi_{\mathrm{dr}}}\right]
\end{aligned}
$$

The derivative of the Lyapunov function becomes:

$$
\dot{\mathrm{V}}=-\mathrm{k}_{\omega_{\mathrm{r}}} \mathrm{e}_{\omega_{\mathrm{r}}}^{2}-\mathrm{k}_{\varphi_{\mathrm{rd}}} \mathrm{e}_{\varphi_{\mathrm{rd}}}^{2}<0
$$

So, the errors in (3) converge to zero.

The state $\varphi_{\text {rd }}$ is unknown, so we need to use his estimation in (8), so the new expression:

$$
\begin{aligned}
& \hat{i}_{\mathrm{sq}}^{*}=\frac{1}{\hat{\varphi}_{\mathrm{rd}} \cdot \mathrm{a}_{6}}\left[\mathrm{k}_{\omega_{\mathrm{r}}} \mathrm{e}_{\omega_{\mathrm{r}}}+\dot{\omega}_{\mathrm{r}}^{*}+\mathrm{a}_{7} \cdot \omega_{\mathrm{r}}+\mathrm{a}_{8} \cdot \mathrm{T}_{1}+\dot{\mathrm{z}}_{\omega_{\mathrm{r}}}\right] \\
& \hat{i}_{\mathrm{sd}}^{*}=\frac{1}{\mathrm{a}_{4}}\left[\mathrm{k}_{\varphi_{\mathrm{rd}}} \mathrm{e}_{\varphi_{\mathrm{rd}}}+\dot{\varphi}_{\mathrm{rd}}^{*}+\mathrm{a}_{5} \hat{\varphi}_{\mathrm{rd}}+\dot{\mathrm{z}}_{\varphi_{\mathrm{rd}}}\right]
\end{aligned}
$$

\section{b) Second step "currents loop"}

Once virtuals inputs are calculated, we define the error on the currents as follows:

$$
\begin{aligned}
& \mathrm{e}_{\mathrm{i}_{\mathrm{sq}}}=\hat{\mathrm{i}}_{\mathrm{sq}}^{*}-\mathrm{i}_{\mathrm{sq}}+\mathrm{z}_{\mathrm{i}_{\mathrm{sq}}} \\
& \mathrm{e}_{\mathrm{i}_{\mathrm{sd}}}=\hat{\mathrm{i}}_{\mathrm{sd}}^{*}-\mathrm{i}_{\mathrm{sd}}+\mathrm{z}_{\mathrm{isd}}
\end{aligned}
$$

With:

$$
\begin{aligned}
& \mathrm{z}_{\mathrm{i}_{\mathrm{sq}}}=\mathrm{k}_{\mathrm{i}_{\mathrm{sq}}}^{\prime} \int_{0}^{\mathrm{t}}\left(\mathrm{i}_{\mathrm{sq}}^{*}-\mathrm{i}_{\mathrm{sq}}\right) \mathrm{dt} \\
& \mathrm{z}_{\mathrm{i}_{\mathrm{sd}}}=\mathrm{k}_{\mathrm{i}_{\mathrm{sd}}}^{\prime} \int_{0}^{\mathrm{t}}\left(\mathrm{i}_{\mathrm{sd}}^{*}-\mathrm{i}_{\mathrm{sd}}\right) \mathrm{dt}
\end{aligned}
$$

Then, the error dynamical equations are expressed by:

$$
\begin{aligned}
& \mathrm{e}_{\mathrm{i}_{\mathrm{sq}}}=\frac{1}{\hat{\varphi}_{\mathrm{rd}} \cdot \mathrm{a}_{6}}\left[\mathrm{k}_{\omega_{\mathrm{r}}} \mathrm{e}_{\omega_{\mathrm{r}}}+\dot{\omega}_{\mathrm{r}}^{*}+\mathrm{a}_{7} \cdot \omega_{\mathrm{r}}+\mathrm{a}_{8} \cdot \mathrm{T}_{1}+\dot{\mathrm{z}}_{\omega_{\mathrm{r}}}\right]-\mathrm{i}_{\mathrm{sq}}+\mathrm{z}_{\mathrm{i}_{\mathrm{sq}}} \\
& \mathrm{e}_{\mathrm{i}_{\mathrm{sd}}}=\frac{1}{\mathrm{a}_{4}}\left[\mathrm{k}_{\varphi_{\mathrm{rdd}}} \mathrm{e}_{\varphi_{\mathrm{rd}}}+\dot{\varphi}_{\mathrm{rd}}^{*}+\mathrm{a}_{5} \hat{\varphi}_{\mathrm{rd}}+\dot{\mathrm{z}}_{\varphi_{\mathrm{dr}}}\right]-\mathrm{i}_{\mathrm{sd}}+\mathrm{z}_{\mathrm{i}_{\mathrm{sd}}}
\end{aligned}
$$

Then, the error equations (5) can be expressed as:

$$
\begin{aligned}
& \dot{\mathrm{e}}_{\omega_{\mathrm{r}}}=-\mathrm{k}_{\omega_{\mathrm{r}}} \mathrm{e}_{\omega_{\mathrm{r}}}+\mathrm{a}_{6} \cdot \hat{\varphi}_{\mathrm{rd}} \cdot \mathrm{e}_{\mathrm{isq}}-\mathrm{a}_{6} \cdot \hat{\varphi}_{\mathrm{rd}} \cdot \mathrm{z}_{\mathrm{i}_{\mathrm{sq}}} \\
& \dot{\mathrm{e}}_{\varphi_{\mathrm{rd}}}=-\mathrm{k}_{\varphi_{\mathrm{dr}}} \mathrm{e}_{\varphi_{\mathrm{dr}}}+\mathrm{a}_{4} \cdot \mathrm{e}_{\mathrm{isd}}-\mathrm{a}_{5} \cdot \tilde{\varphi}_{\mathrm{rd}}-\mathrm{a}_{4} \cdot \mathrm{z}_{\mathrm{i}_{\mathrm{sd}}}
\end{aligned}
$$


Where the flux estimation error is:

$$
\tilde{\varphi}_{\mathrm{rd}}=\hat{\varphi}_{\mathrm{rd}}-\varphi_{\mathrm{rd}}
$$

As well, the dynamical equations for the error $\mathrm{e}_{\mathrm{i}_{\mathrm{sq}}}, \mathrm{e}_{\mathrm{i}_{\mathrm{sd}}}$ can be expressed as:

$$
\begin{aligned}
& \dot{\mathrm{e}}_{\mathrm{i}_{\mathrm{sq}}}=\dot{\hat{\mathrm{i}}}_{\mathrm{sq}}^{*}-\dot{\mathrm{i}}_{\mathrm{sq}}+\dot{\mathrm{z}}_{\mathrm{i}_{\mathrm{sq}}} \\
& \dot{\mathrm{e}}_{\mathrm{i}_{\mathrm{sq}}}=\psi_{\mathrm{i}_{\mathrm{sq}}}-\lambda_{\mathrm{i}_{\mathrm{sq}}}-\mathrm{bv} \mathrm{v}_{\mathrm{sq}}+\dot{\mathrm{z}}_{\mathrm{i}_{\mathrm{sq}}}
\end{aligned}
$$

And

$$
\begin{aligned}
& \dot{\mathrm{e}}_{\mathrm{i}_{\mathrm{sd}}}=\dot{\hat{\mathrm{i}}}_{\mathrm{sd}}^{*}-\dot{\mathrm{i}}_{\mathrm{sd}}+\dot{\mathrm{z}}_{\mathrm{i}_{\mathrm{sd}}} \\
& \dot{\mathrm{e}}_{\mathrm{i}_{\mathrm{sd}}}=\psi_{\mathrm{i}_{\mathrm{sd}}}-\lambda_{\mathrm{i}_{\mathrm{sd}}}-\frac{\mathrm{k}_{\varphi_{\mathrm{rd}}}}{\mathrm{L}_{\mathrm{m}}} \tilde{\varphi}_{\mathrm{rd}}-\mathrm{bv} \mathrm{v}_{\mathrm{sd}}+\dot{\mathrm{z}}_{\mathrm{i}_{\mathrm{sd}}}
\end{aligned}
$$

Where:

$$
\begin{aligned}
& \psi_{\mathrm{i}_{\mathrm{sq}}}=\frac{1}{\hat{\hat{\varphi}}_{\mathrm{rd}} \cdot \mathrm{a}_{6}}\left[\mathrm{k}_{\omega_{\mathrm{r}}} \mathrm{e}_{\omega_{\mathrm{r}}}+\dot{\omega}_{\mathrm{r}}^{*}+\mathrm{a}_{7} \cdot \omega_{\mathrm{r}}+\mathrm{a}_{8} \cdot \mathrm{T}_{1}+\dot{\mathrm{z}}_{\omega_{\mathrm{r}}}\right]+\frac{1}{\hat{\varphi}_{\mathrm{rd}} \cdot \mathrm{a}_{6}}\left[\mathrm{k}_{\omega_{\mathrm{r}}} \dot{\mathrm{e}}_{\omega_{\mathrm{r}}}+\ddot{\omega}_{\mathrm{r}}^{*}+\mathrm{a}_{7} \cdot \dot{\omega}_{\mathrm{r}}+\mathrm{a}_{8} \cdot \dot{\mathrm{T}}_{1}+\ddot{\mathrm{z}}_{\omega_{\mathrm{r}}}\right] \\
& \psi_{\mathrm{i}_{\mathrm{sd}}}=\frac{1}{\mathrm{a}_{4}}\left[\mathrm{k}_{\varphi_{\mathrm{rd}}} \dot{\mathrm{e}}_{\varphi_{\mathrm{rd}}}+\ddot{\varphi}_{\mathrm{rd}}^{*}+\mathrm{a}_{5} \dot{\hat{\varphi}}_{\mathrm{rd}}+\ddot{\mathrm{z}}_{\varphi_{\mathrm{rd}}}\right] \\
& \lambda_{\mathrm{i}_{\mathrm{sq}}}=-\omega_{\mathrm{s}} \cdot \mathrm{i}_{\mathrm{sd}}+\mathrm{a}_{1} \mathrm{i}_{\mathrm{sq}}-\mathrm{a}_{3} \cdot \varphi_{\mathrm{rd}} \cdot \omega_{\mathrm{r}}+\mathrm{a}_{2} \cdot \varphi_{\mathrm{rq}} \\
& \lambda_{\mathrm{i}_{\mathrm{sd}}}=\mathrm{a}_{1} \dot{\mathrm{i}}_{\mathrm{sd}}+\omega_{\mathrm{s}} \cdot \mathrm{i}_{\mathrm{sq}}+\mathrm{a}_{2} \cdot \varphi_{\mathrm{rd}}+\mathrm{a}_{3} \omega_{\mathrm{r}} \cdot \varphi_{\mathrm{rq}}
\end{aligned}
$$

Now we extend the Lyapunov function in (6) to include the new states variables $\mathrm{e}_{\mathrm{i}_{\mathrm{sq}}}, \mathrm{e}_{\mathrm{i}_{\mathrm{sq}}}$ and the parameter estimation errors $\tilde{\varphi}_{\text {rd }}$ as:

$$
\mathrm{V}=\frac{1}{2}\left(\mathrm{e}_{\omega_{\mathrm{r}}}^{2}+\mathrm{e}_{\varphi_{\mathrm{rdd}}}^{2}+\mathrm{e}_{\mathrm{i}_{\mathrm{sq}}}^{2}+\mathrm{e}_{\mathrm{i}_{\mathrm{sd}}}^{2}+\frac{1}{\gamma} \tilde{\varphi}_{\mathrm{rd}}^{2}\right)
$$

Where $\gamma$ is a positive constant known as the adaptive gain. We use the energy function $\mathrm{V}$, to obtain both the control and estimation algorithms.

The derivative of the above equation is:

$$
\dot{\mathrm{V}}=\mathrm{e}_{\omega_{\mathrm{r}}} \dot{\mathrm{e}}_{\omega_{\mathrm{r}}}+\mathrm{e}_{\varphi_{\mathrm{rd}}} \dot{\mathrm{e}}_{\varphi_{\mathrm{rdd}}}+\mathrm{e}_{\mathrm{i}_{\mathrm{sq}}} \dot{\mathrm{e}}_{\mathrm{i}_{\mathrm{sq}}}+\mathrm{e}_{\mathrm{i}_{\mathrm{sd}}} \dot{\mathrm{e}}_{\mathrm{i}_{\mathrm{sd}}}+\frac{1}{\gamma} \tilde{\varphi}_{\mathrm{rd}} \dot{\tilde{\varphi}}_{\mathrm{rd}}
$$

With:

$$
\begin{aligned}
& \dot{\tilde{\varphi}}_{\mathrm{rd}}=\dot{\hat{\varphi}}_{\mathrm{rd}}-\dot{\varphi}_{\mathrm{rd}} \\
& \dot{\tilde{\varphi}}_{\mathrm{rd}}=\dot{\hat{\varphi}}_{\mathrm{rd}}-\left(\mathrm{a}_{4} \cdot \dot{\mathrm{i}}_{\mathrm{sd}}-\mathrm{a}_{5} \cdot \varphi_{\mathrm{rd}}\right)
\end{aligned}
$$


By replacing systems (11), (12) and (13) in (15) equation is obtained:

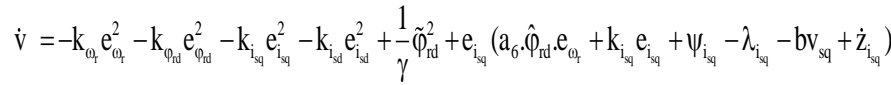

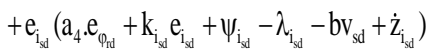

$$
\begin{aligned}
& +\tilde{\varphi}_{\mathrm{rd}}\left[-\mathrm{a}_{5} \cdot \mathrm{e}_{\varphi_{\mathrm{rdd}}}-\frac{\mathrm{k}_{\varphi_{\mathrm{P}}}}{\mathrm{L}_{\mathrm{m} 5}} \mathrm{e}_{\mathrm{i}_{\mathrm{d} d}}+\frac{1}{\gamma}\left(\dot{\hat{\varphi}}_{\mathrm{rd}}-\mathrm{a}_{4} \cdot \mathrm{i}_{\mathrm{sd}}+\mathrm{a}_{5} \cdot \hat{\varphi}_{\mathrm{rd}}\right)\right]
\end{aligned}
$$

For the condition that $\dot{\mathrm{v}}<0$, the control voltages $\mathrm{v}_{\mathrm{sd}}$ and $\mathrm{v}_{\mathrm{sq}}$ as follows:

$$
\begin{aligned}
& \mathrm{v}_{\mathrm{sd}}=\sigma \mathrm{L}_{\mathrm{s}}\left(\mathrm{k}_{\mathrm{i}_{\mathrm{sd}}} \mathrm{e}_{\mathrm{i}_{\mathrm{sd}}}+\psi_{\mathrm{i}_{\mathrm{sd}}}-\lambda_{\mathrm{i}_{\mathrm{sd}}}-\mathrm{bv}_{\mathrm{sd}}+\dot{\mathrm{z}}_{\mathrm{i}_{\mathrm{sd}}}\right) \\
& \mathrm{v}_{\mathrm{sq}}=\sigma \mathrm{L}_{\mathrm{s}}\left(\mathrm{k}_{\mathrm{i}_{\mathrm{sq}}} \mathrm{e}_{\mathrm{i}_{\mathrm{sq}}}+\psi_{\mathrm{i}_{\mathrm{sq}}}-\lambda_{\mathrm{i}_{\mathrm{sq}}}-\mathrm{bv} \mathrm{v}_{\mathrm{sq}}+\dot{\mathrm{z}}_{\mathrm{i}_{\mathrm{sq}}}\right)
\end{aligned}
$$

And the adaptation law of rotor flux $\hat{\varphi}_{\text {rd }}$ given by:

$$
\dot{\hat{\varphi}}_{\mathrm{rd}}=-\gamma\left(-\mathrm{a}_{5} \cdot \mathrm{e}_{\varphi_{\mathrm{rd}}}-\frac{\mathrm{k}_{\varphi_{\mathrm{rd}}}}{\mathrm{L}_{\mathrm{m} 5}} \mathrm{e}_{\mathrm{i}_{\mathrm{sd}}}\right)+\mathrm{a}_{4} \cdot \mathrm{i}_{\mathrm{sd}}-\mathrm{a}_{5} \cdot \hat{\varphi}_{\mathrm{rd}}
$$

\section{ROTOR SPEED MRAS OBSERVER}

\subsection{Principle of MRAS observer}

Currently there are many techniques for achieving speed estimator, but the most used is certainly the approach using an Model Reference Adaptive System (MRAS), this for its easier implementation and its good performance in terms of precision and stability [22].

The technique of MRAS based on the rotor flux for estimating the speed of the induction machine, originally developed by C. Schauder [23], is certainly the most popular strategy. The principle of the speed estimation by a MRAS is based on the comparison of rotor flux outputs of two estimators using different formulations. The first estimator is based on the stator equations defining a voltage model, called the reference model and the second, named adjustable or adaptive model is described by the rotor equations, it defines a current model.

The estimated components of the rotor flux, for both models, are based only on the measurement of stator quantities. We usere the reference voltages and the measured currents usually expressed in a stationary reference frame related to the stator $(\alpha-\beta)$ [5]. The structure of the adaptive observer is shown in Figure 1.

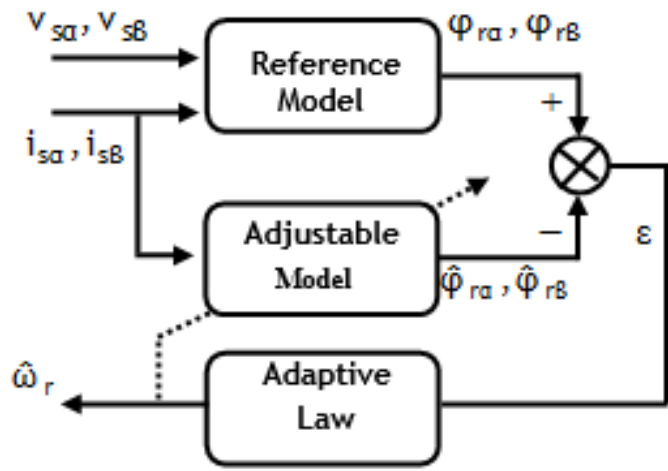

Figure 1. Block diagram of MRAS observer 


\subsection{Estimation of rotor speed by MRAS observer}

The equations of the reference model are given by:

$$
\left\{\begin{array}{l}
\dot{\varphi}_{\mathrm{r} \alpha}=\frac{\mathrm{L}_{\mathrm{r}}}{\mathrm{L}_{\mathrm{m}}}\left(\mathrm{v}_{\mathrm{s} \alpha}-\mathrm{R}_{\mathrm{s}} \mathrm{i}_{\mathrm{s} \alpha}-\sigma \mathrm{L}_{\mathrm{s}} \dot{i}_{\mathrm{s} \alpha}\right) \\
\dot{\varphi}_{\mathrm{r} \beta}=\frac{\mathrm{L}_{\mathrm{r}}}{\mathrm{L}_{\mathrm{m}}}\left(\mathrm{v}_{\mathrm{s} \beta}-\mathrm{R}_{\mathrm{s}} \mathrm{i}_{\mathrm{s} \beta}-\sigma \mathrm{L}_{\mathrm{s} s \beta} \dot{\mathrm{i}}_{\mathrm{s}}\right)
\end{array}\right.
$$

The equations of the adjustable model are given by:

$$
\left\{\begin{array}{l}
\dot{\hat{\varphi}}_{\mathrm{r} \alpha}=-\frac{1}{\mathrm{~T}_{\mathrm{r}}} \hat{\varphi}_{\mathrm{r} \alpha}-\omega_{\mathrm{r}} \hat{\varphi}_{\mathrm{r} \beta}+\frac{\mathrm{L}_{\mathrm{m}}}{\mathrm{T}_{\mathrm{r}}} \mathrm{i}_{\mathrm{s} \alpha} \\
\dot{\hat{\varphi}}_{\mathrm{r} \beta}=-\frac{1}{\mathrm{~T}_{\mathrm{r}}} \hat{\varphi}_{\mathrm{r} \beta}+\omega_{\mathrm{r}} \hat{\varphi}_{\mathrm{r} \alpha}+\frac{\mathrm{L}_{\mathrm{m}}}{\mathrm{T}_{\mathrm{r}}} \mathrm{i}_{\mathrm{s} \beta}
\end{array}\right.
$$

With:

$$
\mathrm{T}_{\mathrm{r}}=\frac{\mathrm{L}_{\mathrm{r}}}{\mathrm{R}_{\mathrm{r}}}
$$

We notice that the rotor speed is only present in equations (19) and not in equations (18). The adaptation algorithm is chosen to make the adjustable model converge toward the reference model by minimizing the error. For this, the algorithm parameters are defined according to the criterion of hyperstability of Popov. [23]

The error between the states of the two models can be expressed in matrix form by:

$$
\left[\begin{array}{c}
\varepsilon_{\alpha} \\
\varepsilon_{\beta}
\end{array}\right]=\left[\begin{array}{c}
\varphi_{\mathrm{r} \alpha}-\hat{\varphi}_{\mathrm{r} \alpha} \\
\varphi_{\mathrm{r} \beta}-\hat{\varphi}_{\mathrm{r} \beta}
\end{array}\right]
$$

And from equations (18) and (19) its dynamics are given by:

$$
\dot{\varepsilon}=\mathrm{A} . \varepsilon-\mathrm{W}
$$

With:

$$
\dot{\varepsilon}=\left[\begin{array}{c}
\dot{\varepsilon}_{\alpha} \\
\dot{\varepsilon}_{\beta}
\end{array}\right], A=\left[\begin{array}{c}
-\frac{1}{\mathrm{~T}_{\mathrm{r}}}-\omega_{\mathrm{r}} \\
\omega_{\mathrm{r}}-\frac{1}{\mathrm{~T}_{\mathrm{r}}}
\end{array}\right], \varepsilon=\left[\begin{array}{l}
\varepsilon_{\alpha} \\
\varepsilon_{\beta}
\end{array}\right], \mathrm{W}=\left(\omega_{\mathrm{r}}-\hat{\omega}_{\mathrm{r}}\right) \cdot\left[\begin{array}{c}
-\hat{\varphi}_{\mathrm{r} \alpha} \\
\hat{\varphi}_{\mathrm{r} \beta}
\end{array}\right]
$$

Schauder [24] offers an adaptation law that meets the criterion of Popov and given by equation:

$$
\hat{\omega}_{\mathrm{r}}=\chi_{2}(\varepsilon)+\int_{0}^{\mathrm{t}} \chi_{1}(\varepsilon) \mathrm{d} \tau
$$

The criterion of Popov requires satisfaction of the following inequality [23]:

$$
\int_{0}^{\mathrm{t}} \varepsilon^{\mathrm{T}} \mathrm{Wdt} \geq-\mu^{2}
$$


With $\mu$ is a positive constant.

Replacing $\varepsilon$ and $\mathrm{W}$ by their values, Equation (23) becomes:

$$
\int_{0}^{t}\left\{\left[\varepsilon_{\alpha} \hat{\varphi}_{\mathrm{r} \beta}-\varepsilon_{\beta} \hat{\varphi}_{\mathrm{r} \alpha}\right] \cdot\left[\omega_{\mathrm{r}}-\chi_{2}(\varepsilon)-\int_{0}^{\mathrm{t}} \chi_{1}(\varepsilon) \mathrm{d} \tau\right]\right\} \geq-\mu^{2}
$$

We can solve the equation (24) using the following relationship:

$$
\int_{0}^{\mathrm{t}} \mathrm{k}\left(\frac{\mathrm{d}}{\mathrm{dt}} \mathrm{f}(\mathrm{t})\right) \mathrm{f}(\mathrm{t}) \mathrm{dt} \geq-\frac{1}{2} \mathrm{kf}(0)^{2}
$$

The use of the above expression provides the following functions:

$$
\begin{aligned}
& \chi_{1}=\mathrm{k}_{\mathrm{i}}\left(\left(\varepsilon_{\beta} \hat{\varphi}_{\mathrm{r} \alpha}-\varepsilon_{\alpha} \hat{\varphi}_{\mathrm{r} \beta}\right)=\mathrm{k}_{\mathrm{i}}\left(\varphi_{\mathrm{r} \beta} \hat{\varphi}_{\mathrm{r} \alpha}-\varphi_{\mathrm{r} \alpha} \hat{\varphi}_{\mathrm{r} \beta}\right)\right. \\
& \chi_{2}=\mathrm{k}_{\mathrm{p}}\left(\varepsilon_{\beta} \hat{\varphi}_{\mathrm{r} \alpha}-\varepsilon_{\alpha} \hat{\varphi}_{\mathrm{r} \beta}\right)=\mathrm{k}_{\mathrm{p}}\left(\varphi_{\mathrm{r} \beta} \hat{\varphi}_{\mathrm{r} \alpha}-\varphi_{\mathrm{r} \alpha} \hat{\varphi}_{\mathrm{r} \beta}\right)
\end{aligned}
$$

Replacing the equations (26) in equation (22) there was obtained:

$$
\hat{\omega}_{\mathrm{r}}=\mathrm{k}_{\mathrm{p}}\left(\varphi_{\mathrm{r} \beta} \hat{\varphi}_{\mathrm{r} \alpha}-\varphi_{\mathrm{r} \alpha} \hat{\varphi}_{\mathrm{r} \beta}\right)+\mathrm{k}_{\mathrm{i}} \int\left(\varphi_{\mathrm{r} \beta} \hat{\varphi}_{\mathrm{r} \alpha}-\varphi_{\mathrm{r} \alpha} \hat{\varphi}_{\mathrm{r} \beta}\right) \mathrm{dt}
$$

$\mathrm{k}_{\mathrm{p}}$ and $\mathrm{k}_{\mathrm{i}}$ are the PI controller gains of the adaptation mechanism.

The flux position and the Park angle is determined by:

$$
\hat{\theta}_{\mathrm{s}}=\operatorname{arctg}\left(\frac{\hat{\varphi}_{\mathrm{r} \beta}}{\hat{\varphi}_{\mathrm{r} \alpha}}\right)
$$

The integral Backstepping control with the MRAS-Backstepping observer combination to estimate the rotor flux and the rotor speed for induction machine can be presented by the block diagram of Figure 2 .

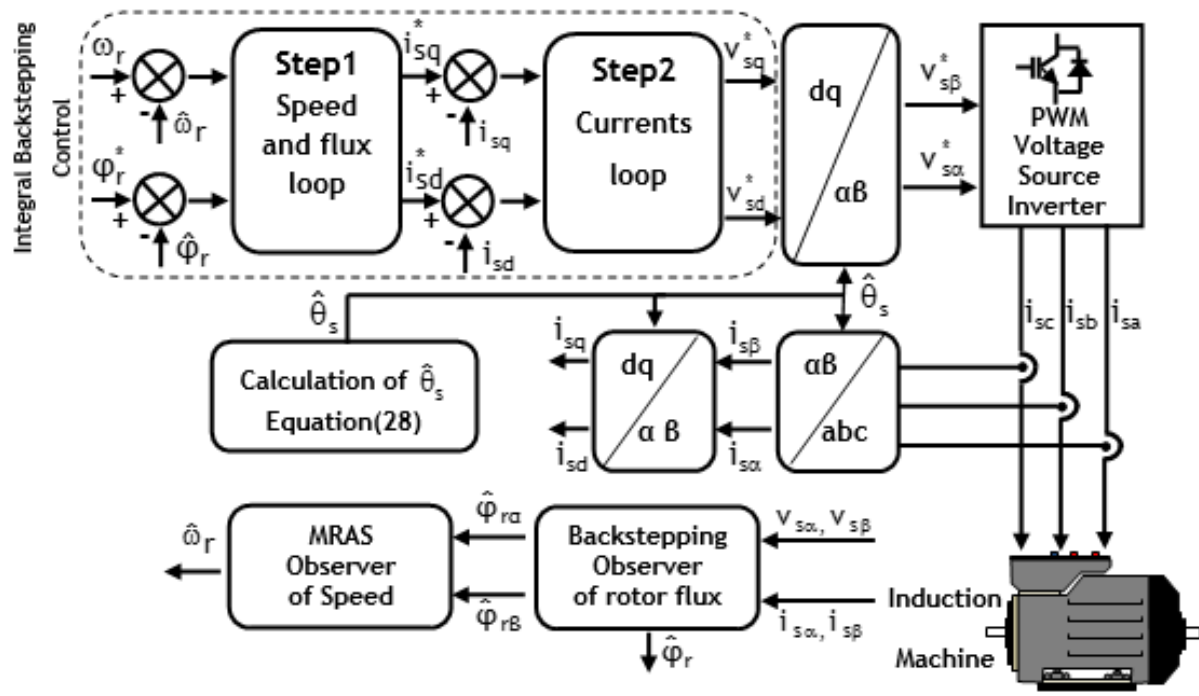

Figure 2. Block diagram of Sensorless integral Backstepping control scheme associated with MRAS-Backstepping observer

\section{SIMULATION RESULTS AND INTERPRETATIONS}

MRAS-based Sensorless Speed Integral Backstepping Control for Induction Machine ... (Mohamed Horch) 


\subsection{Simulation results}

To evaluate the performance of the control and the two observers proposed in this study, two Benchmarks are proposed by [3]. The first Benchmark presents rapid transitions and operating areas at large and low speeds, the second Benchmark consists of low and very low speed operation and inversion.

The objective is to impose reference trajectories taking into account the problems of observability of induction machine at very low speed. Recall that the sufficient condition of observability loss corresponds to having simultaneously constant mechanical speed and zero stator pulsation [25].

The results presented below were obtained by simulation tests conducted for sensorless integral Backstepping control scheme associated with the MRAS and Backstepping observer, on the profiles imposed by Benchmarks 1 and 2.

a) Benchmarks "1"

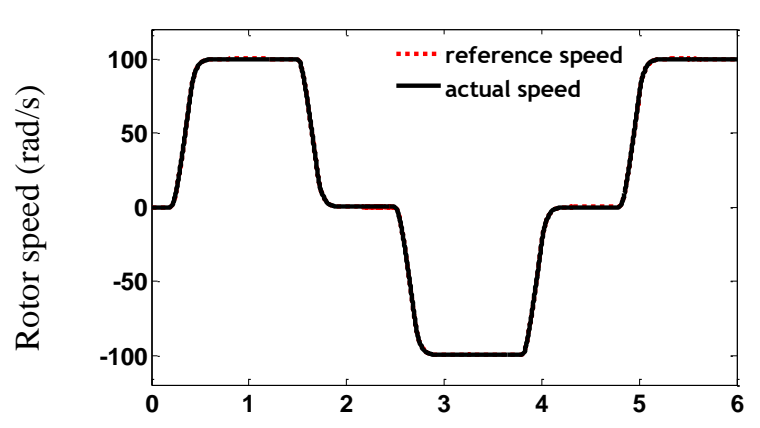

Time (sec.)
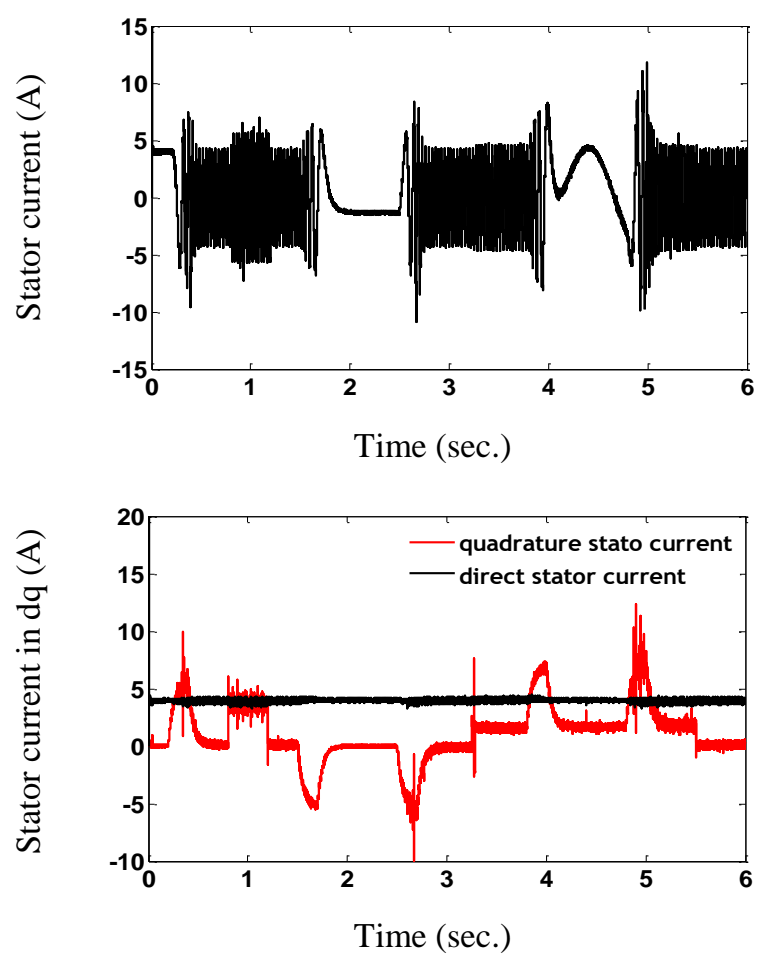
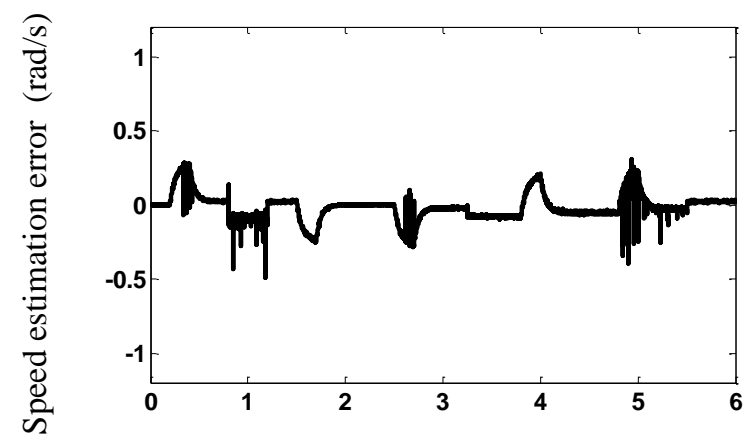

Time (sec.)
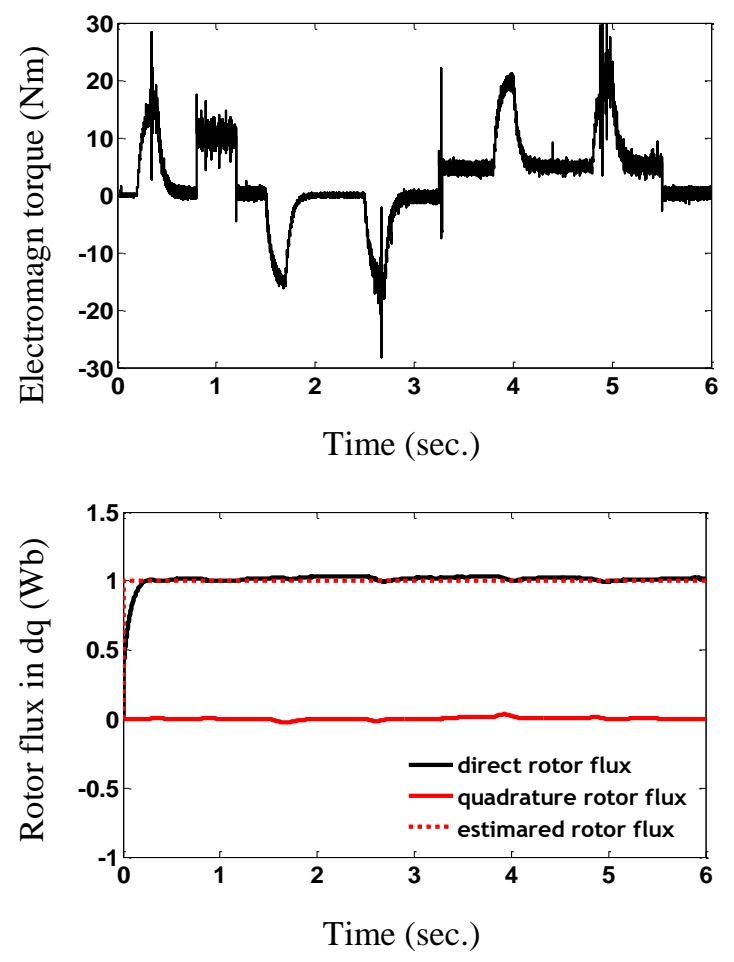


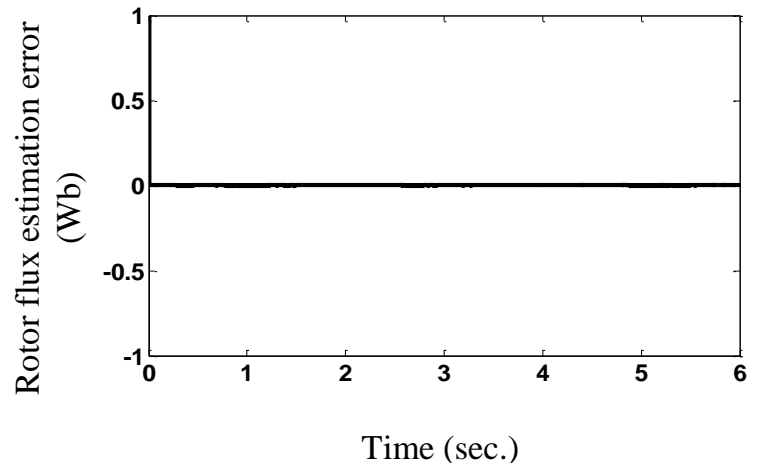

Figure 3. Performance in Benchmarks 1 of the proposed observer during starting operation with load change

b) Benchmarks "2"
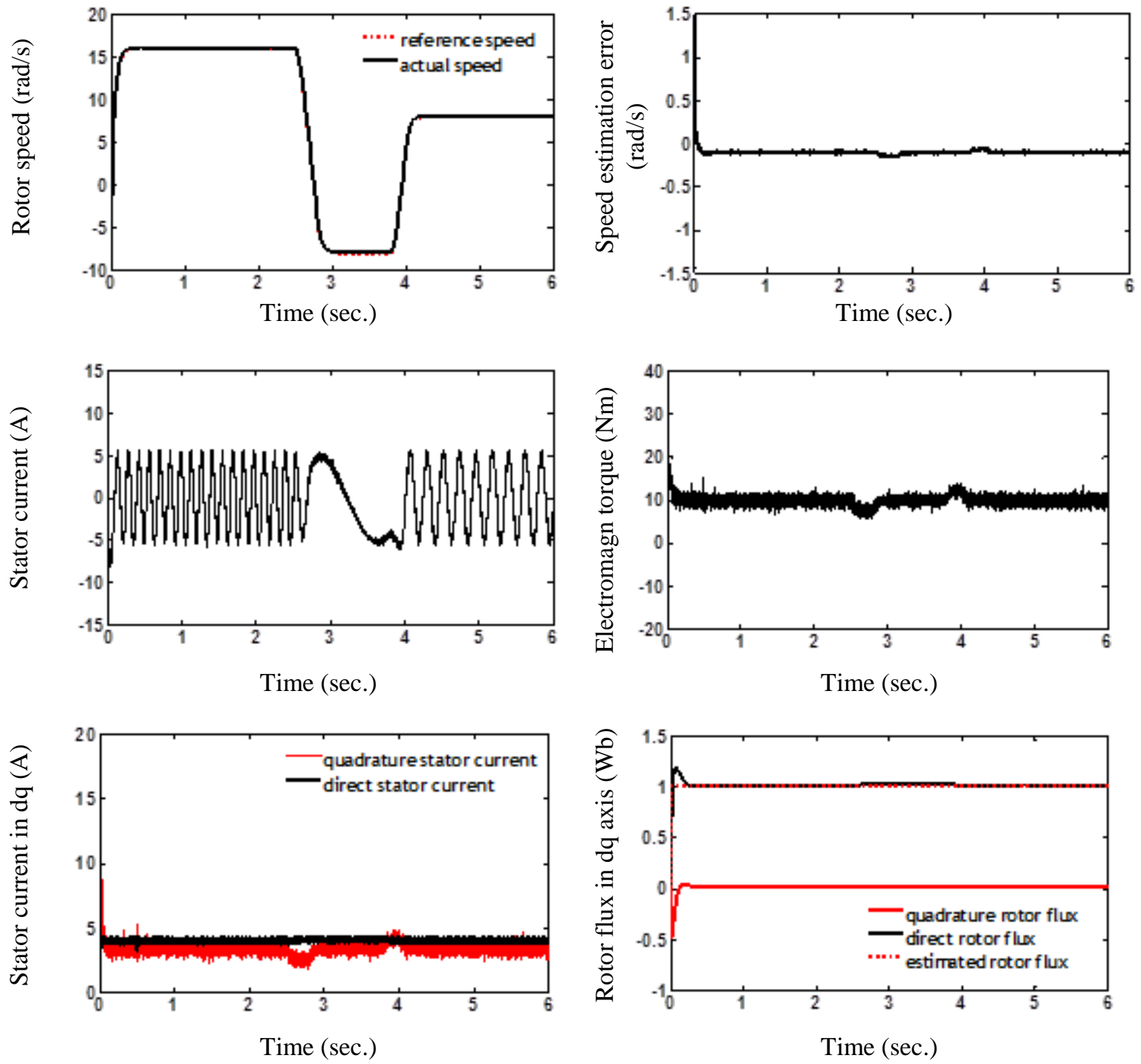


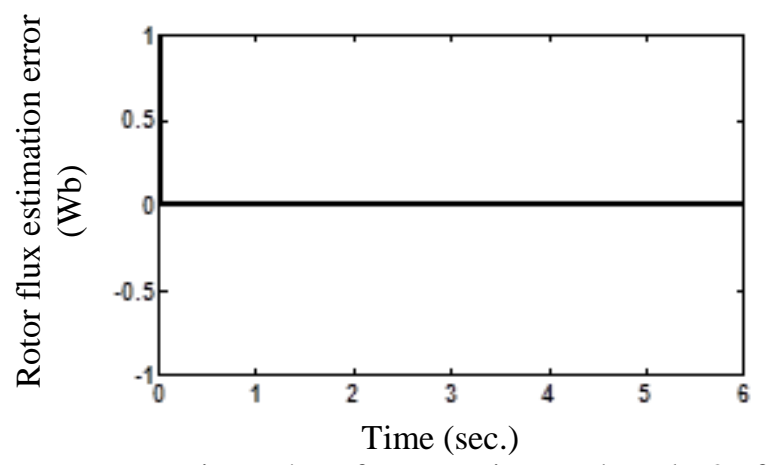

Figure 4.Performance in Benchmarks 2 of the proposed observer at low speed region

\subsection{Interpretations}

Benchmark 1 defines the reference trajectories for speed, rotor flux and load torque, as shown in the figure 3. At the beginning, reference speed is imposed zero, to enable the flux to reach its nominal value of $1 \mathrm{~Wb}$ which is then kept constant. At $\mathrm{t}=0,2 \mathrm{~s}$, the rotor speed is increased to $100 \mathrm{rad} / \mathrm{s}$, according to an acceleration ramp, and remains constant up to $1,5 \mathrm{~s}$. Then it is reduced, reaching a value of zero at $1,7 \mathrm{~s}$, the machine is then maintained at standstill until 2,5 s. This first phase is used to test the behavior of the sensorless control for speed variations in quick transitions, and for a first critical area of operation, between $1,7 \mathrm{~s}$ and $2,5 \mathrm{~s}$, at zero speed and without mechanical load. The application and removal of a torque load at the moments $0,8 \mathrm{~s}$ and $1,2 \mathrm{~s}$ will also assess the impact of this type of disturbance. Then, between $2,5 \mathrm{~s}$ and $4,8 \mathrm{~s}$, a quasi-symmetric velocity profile is imposed in the opposite rotational direction, defining a second area of critical operating at a rate of $-3,25 \mathrm{rad} / \mathrm{s}$ between $4 \mathrm{~s}$ and 4,8 s. Finally, the machine speed is again increased to $100 \mathrm{rad} / \mathrm{s}$, to be there maintained up to $6 \mathrm{~s}$. During the second critical phase, a torque load equal to $50 \%$ of the nominal value is applied at time $3,25 \mathrm{~s}$ and canceled at $5,5 \mathrm{~s}$, with the aim of analyzing the behavior of the training in an unobservable area, but this time in the presence of a mechanical load. The objective of Benchmark 2 is to evaluate the performance strategies of sensorless control at low and very low speed sensor in conditions of unobservable area of the machine and to highlight the influence of the load torque on the behavior of the control.

In figure 3, we notice that the estimated speed perfectly follows the machine speed with good behavior in terms of tracking and disturbance rejection. The tracking error is minimal never exceed $1 \mathrm{rad} / \mathrm{s}$ during transient. The estimated speed converges quickly and accurately to the actual machine speed and the estimation error is also small, but presents some oscillations between $1,25 \mathrm{rad} / \mathrm{s}$ to $1,40 \mathrm{rad} / \mathrm{s}$, during motor start. When going from 100 to $0 \mathrm{rad} / \mathrm{sec}$, then -100 to $0 \mathrm{rad} / \mathrm{s}$, this error is 0,3 and $0,4 \mathrm{rad} / \mathrm{s}$, while in the two critical areas the maximum values are 0,050 and $-0,040 \mathrm{rad} / \mathrm{s}$ for steady state. The decoupling is adequately maintained. However, we observe small oscillations during transients and very low orientation errors in the two critical areas.

Figure 4 shows clearly that the estimated rotor speed follows with excellent precision the actual machine speed. The estimation error is very small at low and very low speed steady. When reversing the direction of rotation from $-8 \mathrm{rad} / \mathrm{s}$ to $8 \mathrm{rad} / \mathrm{s}$, the estimation shows some oscillations with a maximum error of $0,4 \mathrm{rad} / \mathrm{s}$, which is acceptable.

\section{CONCLUSION}

In this paper, a new sensorless drive system approach is presented, coupled with a direct vector control for induction machine. The proposed method consists on the use of integral backstepping control with a nonlinear observer of rotor flux and adaptive observer for estimating rotor speed.

The results show excellent estimation under different operating conditions, especially at low speeds. In addition, it shows robustness against external disturbances and changes in machine parameters. This study shows that it is possible to realize an association of two observers for sensorless control based on a nonlinear approach with a high level of performance.

\section{NOMENCLATURE}

$\mathrm{T}_{\mathrm{em}}, \mathrm{T}_{\mathrm{L}}$ : Electromagnetic and load torque. 
$\mathrm{i}_{\mathrm{sd}}, \mathrm{i}_{\mathrm{sq}}:$ Stator currents.

$\varphi_{\text {rd }}, \varphi_{\text {rq }}:$ Rotor flux.

$\omega_{\mathrm{m}}$ : Electrical angular.

$\sigma:$ Total leakage factor.

$\mathrm{R}_{\mathrm{s}}$ and $\mathrm{R}_{\mathrm{r}}$ : Stator and rotor resistances.

$\mathrm{L}_{\mathrm{s}}$ and $\mathrm{L}_{\mathrm{r}}$ : Stator and rotor inductances.

$\mathrm{L}_{\mathrm{m}}$ : Mutual inductance.

$\mathrm{p}$ : Number of pole pairs.

$\mathrm{J}$ : Moment of inertia.

$\mathrm{f}_{\mathrm{c}}$ : Friction coefficient.

\section{APPENDIX}

Table 1: Nominal parameters of the induction machine used:

\begin{tabular}{l|l}
\hline Rated power & $1.5 \mathrm{~kW}$ \\
\hline Rated voltage & $220 \mathrm{~V}$ \\
\hline Rated speed & $1428 \mathrm{rpm}$ \\
\hline Nominal frequency & $50 \mathrm{~Hz}$ \\
\hline Rated stator current & $3.64 \mathrm{~A}$ \\
\hline Stator resistance & $4.85 \Omega$ \\
\hline Rotor resistance & $3.805 \Omega$ \\
\hline Cyclic stator inductance & $0.274 \mathrm{H}$ \\
\hline Cyclic rotor inductance & $0.274 \mathrm{H}$ \\
\hline Mutual inductance & $0.258 \mathrm{H}$ \\
\hline Number of pole pairs & 2 \\
\hline Moment of inertia & $0.031 \mathrm{~kg} / \mathrm{m}^{2}$ \\
\hline Friction coefficient & $0.00114 \mathrm{Nm} . \mathrm{s} / \mathrm{rd}$ \\
\hline
\end{tabular}

\section{REFERENCES}

[1] J. Norberto, J. Ramon, S. Ortigoza, J. Meza, "Simulation, Construction, and Validation of a DC/DC Buck Power Converter-DC Motor System"International Conference on Electronics, Communications and Computers (CONIELECOMP), pages: 118 - 124, 2016.

[2] M. Horch, A. Boumediene, L.baghli, "Sensorless High-order Super Twisting Sliding modes Vector Control for Induction Motor Drives", 8th International Conference on Modelling, Identification and Control (ICMIC), ICMIC'2016, Algiers, Algeria, 15-17 November 2016.

[3] A.Mechernene, "Apport des techniques de l'intelligence artificielle à la commande sans capteur de vitesse d'une machine asynchrone", PhD thesis, University of Sciences and Technology of Oran (USTO), 2014.

[4] M. Taherzadeh, S. Carriere, M. Joorabian, F. Betin, R. Kianinezhad "Analysis and Control of Six-Phase Induction Machines in Unbalanced Operating Situation due to Phase Opening" IECON 2014 - 40th Annual Conference of the IEEE Industrial Electronics Society, Pages: 417 - 423, 2014.

[5] N.P. Quang, J-A. Dittrich, "Vector control of three-phase AC machines", system development in the practice. Springer Berlin, 2008.

[6] J. Bocker, S. Mathapati, "State of the Art of Induction Motor Control", in Proceedings book of IEEE International Conference on Electric Machines \& Drives, IEMDC '07, vol. 2, pp. 1459-1464, 3-5 May 2007.

[7] F. Malrait, "Problèmes d'identification et d'observabilité du moteur à induction dans la variation de vitesse industrielle sans capteur", Thèse en vue de l'obtention du titre de Docteur de l'Ecole Nationale Supérieure des Mines de Paris, France, soutenue publiquement le 07-02-2001.

[8] F. Mehazzem, A.L. Nemmour, A. Reama, H. Benalla, "Nonlinear integral backstepping control for induction motors, "Electrical Machines and Power Electronics, Electromotion Joint Conference, vol. 36, no. 3, pp. 331 - 336, Sept. 2011.

[9] F. Mehazzem, A. Reama, Y. Hamam, and H. Benalla, "Real time implementation of backstepping controller in indirect field oriented control of induction motor drive", Second International IEEE Conference on Power Engineering, Energy and Electrical Drives, POWERENG, March 2009.

[10] J. Mohanalakshmi, H. N. Suresh, "Sensorless speed estimation and vector control of an Induction Motor drive using model reference adaptive control", International Conference on Power and Advanced Control Engineering (ICPACE), pp.377 - 382, 2015.

MRAS-based Sensorless Speed Integral Backstepping Control for Induction Machine ... (Mohamed Horch) 
[11] T. Orlowska, Kowalska and M. Dybkowski , "Stator Current based MRAS estimator for a wide range speed Sensor less induction motor drives", IEEE Transactions on Industrial Electronics vo1.51, No. 4, April 2010, pp. 1296 1308.

[12] A. Mechernene, M. Zerikat, S. Chekroun, “Adaptive Speed Observer using Artificial Neural Network for Sensorless Vector Control of Induction Motor Drive", Automatika, Journal for Control, Measurement, Electronics, Computing and Communications, Vol. 53, N³, pp. 263-271, July-September 2012.

[13] A.K. Rathore, "Improved Performance of Fuzzy Logic Based Direct Field Oriented Controlled Induction Motor", 9th IEEE International Congress Power Electronics, pp. 152-157, 2008.

[14] L. Baghli, "Contribution à la commande de la machine asynchrone: Utilisation de la logique floue, des réseaux de neurones et des algorithmes génétiques", PhD thesis, University of Henri Poincaré, Nancy-I, 1999.

[15] F. Blaschke, "The principle of field oriented as applied tothe new transvector closed-loop control system for rotatingfieldmachine," Siemens Review XXXIX, vol. 5, no. 4, pp.217-220, 1972.

[16] A.Kechich, B.Mazari, "Application to Adaptive Backstepping for Permanent Magnet Synchronous Machine" Electrotehnica, Electronica, Automatica, vol. 60(2012), nr. 1

[17] O. Boughazi, A. Boumedienne, H. Glaoui, “Sliding Mode Backstepping Control of Induction Motor" International Journal of Power Electronics and Drive System (IJPEDS), vol. 4 (2014), nr. 4

[18] D. Traoré, J. De Leon, A. Glumineau and L. Loron. "Adaptive Interconnected Observer-Based Backstepping Control Design For Sensorless Induction Motor" : Experimental results. Soumis à Control Engineering Practice 2008.

[19] Y. Al-Younes, M. A Jarrah, "Attitude stabilization of quadrotor UAV using Backstepping Fuzzy Logic and Backstepping Least-Mean-Square controllers" Proceeding of the 5th International Symposium on Mechatronics and its Applications (ISMA08) of IEEE, pp. 1-11 Amman, Jordan, May 27-29,2008.

[20] H. Echeikh, R Trabelsi, Atif Iqbal, M.F Mimouni, R Alammari, "Online Adaptation of Rotor Resistance based on Sliding Mode Observer with Backstepping Control of A Five-Phase Induction Motor Drives" International Journal of Power Electronics and Drive System (IJPEDS), vol. 7(2016),nr. 3

[21] M.Horch, A.Boumediene, L.Baghli, "Nonlinear Integral Backstepping Control for Induction Motor drive with Adaptive Speed Observer using SuperTwisting Strategy" Electrotehnica, Electronica, Automatica, vol. 64(2016),nr. 1

[22] C. Schauder, "Model Reference Adaptive System with Simple Sensorless Flux Observer for Induction Motor Drive" IEEE International Conference on Power Electronics, Drives and Energy Systems, pp.1-6, December16-19, 2012, Bengaluru, India.

[23] L. Sbita and M.Ben Hamed, "An MRAS-based full order Luenberger observer for sensorless DRFOC of induction motors", ICGST-ACSE journal, volume 7, issue 1, May 2007.

[24] C. Schauder, "Adaptive speed identification for vector control of induction motors without rotational transducers" IEEE Transactions on Industry Applications, vol. 28, no.5, pp. 1054-1061, 1992.

[25] D.Traore, "Commande non lineaire sans capteur de la machine asynchrone", $\mathrm{PhD}$ thesis, Central School de Nantes, France, 2008. 